\title{
On soil-structure interaction in large non-slender partially buried structures
}

\author{
J. Vega · J. J. Aznárez · A. Santana · E. Alarcón · \\ L. A. Padrón · J. J. Pérez • O. Maeso
}

\begin{abstract}
This paper addresses the seismic analysis of a deeply embedded non-slender structure hosting the pumping unit of a reservoir. The dynamic response in this type of problems is usually studied under the assumption of a perfectly rigid structure using a sub-structuring procedure (three-step solution) proposed specifically for this hypothesis. Such an approach enables a relatively simple assessment of the importance of some key factors influencing the structural response. In this work, the problem is also solved in a single step using a direct approach in which the structure and surrounding soil are modelled as a coupled system with its actual geometry and flexibility. Results indicate that, quite surprisingly, there are significant differences among prediction using both methods. Furthermore, neglecting the flexibility of the structure leads to a significant underestimation of the spectral accelerations at certain points of the structure.
\end{abstract}

Keywords Soil-structure interaction · Direct method · Three-step solution · Buried structures · Boundary Element Method

\section{Introduction}

It is a well known fact that soil-structure interaction (SSI) should be considered in the dynamics of deeply embedded non-slender structures under ground motion. When analysing

\section{J. Vega}

Centro de Modelado en Ingeniería Mecánica (CEMIM-F2I2), Madrid 28006, Spain

\section{J. J. Aznárez · A. Santana · L. A. Padrón · J. J. Pérez · O. Maeso (凶)}

Instituto Universitario de Sistemas Inteligentes y Aplicaciones Numéricas en Ingeniería (SIANI),

Universidad de Las Palmas de Gran Canaria, Las Palmas de Gran Canaria 35017, Spain

e-mail: omaeso@iusiani.ulpgc.es 
the dynamic response of such an structure (for instance large diameter caissons or shaft foundations, and pumping stations) the hypothesis of infinite stiffness is usually assumed. More precisely, embedded structures are considered to be rigid when the relation between depth of embedment and width is smaller than 2, 3 or 4 depending on the authors and application (Mylonakis 2001; Varun et al. 2009), therefore many of these structures are seismically designed following that assumption of rigidness.

The basic methods for the analysis of dynamic soil-structure interaction problems may be classified in direct and sub-structuring methods. Direct methods allow soil and structure to be modelled and analysed simultaneously in a single step. On the contrary, substructuring methods study soil and structure separately and, if the assumption of a perfectly rigid structure is adopted, the method leads to a three-step solution (Kausel and Roesset 1974; Kausel et al. 1978; Villaverde 2009) that allows parametric studies on the factors known to influence the response. However, when the hypothesis of rigidity of the structure foundation does not apply, the use of sub-structuring or direct methods requires soil and structure to be modelled with their actual geometry using more powerful numerical methods, which would imply higher computational effort in both cases. The literature is rich in examples of models addressing soil-structure interaction problems (Kausel 2010) that use either the sub-structuring or the direct approaches in combination with Finite Element Method (FEM) or Boundary Element Method (BEM).

This paper addresses the seismic analysis of a real massive deeply embedded structure, a structural typology traditionally analysed with the three-step approach, the objective being the assessment of the motion at discrete points of the structure consistent with a given free field ground motion. The structure is a part of a big industrial installation and hosts equipment that could be seriously affected during an earthquake. The relation between the embedded length and width of the structure is smaller than 2 , approximately 1.7 , thus it may be considered as a non-slender structure. This study has been performed using sub-structuring and direct methodologies in order to assess the validity of the rigid body assumption, which is the main goal of this paper.

In the first part of the paper the problem at hand is presented. Then, the three-step and direct methods are briefly reviewed. Finally, results obtained with both methods are presented, compared and discussed. Attention will be paid not only to final results, but also to partial ones. The boundary element method is used when applying both methodologies. In each case complex valued relations between response at the points of interest and at free field are calculated. Frequency domain results are later transformed to the time domain using Fourier transform.

\section{Problem description}

Figure 1 shows a geometrical description of the structure under study, which is an $80 \mathrm{~m}$ high almost cylindrical concrete structure, with its lower $50 \mathrm{~m}$ embedded in the soil. The exterior diameter of the non-embedded part of the structure is $28 \mathrm{~m}$. In the embedded length, the thickness of the excavation walls must be added, resulting in a exterior diameter of $30 \mathrm{~m}$. The bottom $20 \mathrm{~m}$ of the structure are composed by a great number of slabs and stiffeners that provide a large rigidity. Therefore, this bottom part, defined as domain 2 (domain 1 corresponds to the rest of the structure) has been considered solid, with the stiffness of the concrete but an equivalent density. Table 1 shows the values of the different properties of both domains of the structure, with $\mu$ being the shear modulus, $v$ the Poisson's ratio, $\rho$ the density, $\xi$ the internal damping coefficient and $c_{s}$ the $\mathrm{S}$ wave propagation velocity. The total 
Fig. 1 Geometrical description of the structure

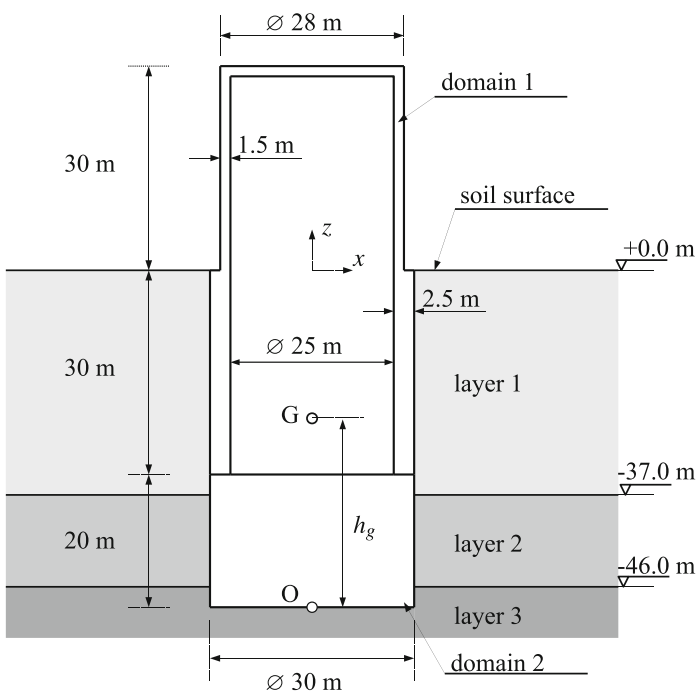

Table 1 Elastic properties of structure and soil layers

\begin{tabular}{llllll}
\hline & Domain 1 & Domain 2 & Soil layer 1 & Soil layer 2 & Soil layer 3 \\
\hline$v$ & 0.2 & 0.2 & 0.3 & 0.3 & 0.3 \\
$\rho$ & $2,685.85 \mathrm{~kg} / \mathrm{m}^{3}$ & $2,253.42 \mathrm{~kg} / \mathrm{m}^{3}$ & $2,000 \mathrm{~kg} / \mathrm{m}^{3}$ & $2,100 \mathrm{~kg} / \mathrm{m}^{3}$ & $2,200 \mathrm{~kg} / \mathrm{m}^{3}$ \\
$\xi$ & 0.05 & 0.05 & 0.05 & 0.05 & 0.05 \\
$\mu$ & $1.15 \times 10^{10} \mathrm{~N} / \mathrm{m}^{2}$ & $1.15 \times 10^{10} \mathrm{~N} / \mathrm{m}^{2}$ & $5 \times 10^{8} \mathrm{~N} / \mathrm{m}^{2}$ & $1.029 \times 10^{9} \mathrm{~N} / \mathrm{m}^{2}$ & $2.2 \times 10^{9} \mathrm{~N} / \mathrm{m}^{2}$ \\
$c_{s}$ & $2,069.23 \mathrm{~m} / \mathrm{s}$ & $2,259.06 \mathrm{~m} / \mathrm{s}$ & $500 \mathrm{~m} / \mathrm{s}$ & $700 \mathrm{~m} / \mathrm{s}$ & $1,000 \mathrm{~m} / \mathrm{s}$ \\
\hline
\end{tabular}

mass $M$ of the structure is $61.3 \times 10^{6} \mathrm{~kg}$, the inertia $I_{G}$ is $3.51 \times 10^{10} \mathrm{~kg} \mathrm{~m}^{2}$ and the distance $h_{g}$ from the base to the center of gravity is $28.36 \mathrm{~m}$. The structure hosts the pumping unit of a large reservoir and other critical devices, such as a crane, that could be seriously affected during an earthquake. For this reason, part of the seismic analyses will focus on the natural frequencies of such devices, estimated to be around 3.89, 7.69 and $12.05 \mathrm{~Hz}$.

The soil is composed of three layers. The top layer is a stiff clay formation and reaches $37 \mathrm{~m}$ depth. The second layer is formed by conglomerates and is $9 \mathrm{~m}$ depth. Finally, the bottom layer is a half space of strongly consolidated detrital sedimentary rock. Only static tests were carried out in the characterization of the different soil layers, so no measurements of $\mathrm{S}$ and $\mathrm{P}$ wave velocities were available. For this reason, some correlations and empirical rules, especially Ishihara (1996) and Seed and Idriss (1970), were used to estimate S wave velocities and soil dynamic shear stiffness. A sensitivity analysis was carried out in order to find out the most conservative soil profile among several ones, all compatible with the field tests results. Having defined the design accelerograms (described below) at the free field surface, the corresponding acceleration response spectra were computed at $-50.0 \mathrm{~m}$ (using SHAKE (Schabel et al. 1972) to obtain the accelerogram at such depth). The profile in Fig. 2 was finally selected because it leads to higher (conservative) values for the range of frequencies of interest in regard to the hosted equipment. All results shown in this paper will be based upon it. Table 1 summarizes the properties finally used to characterize the different soil layers. 
Fig. 2 Shear wave velocity profile for the soil
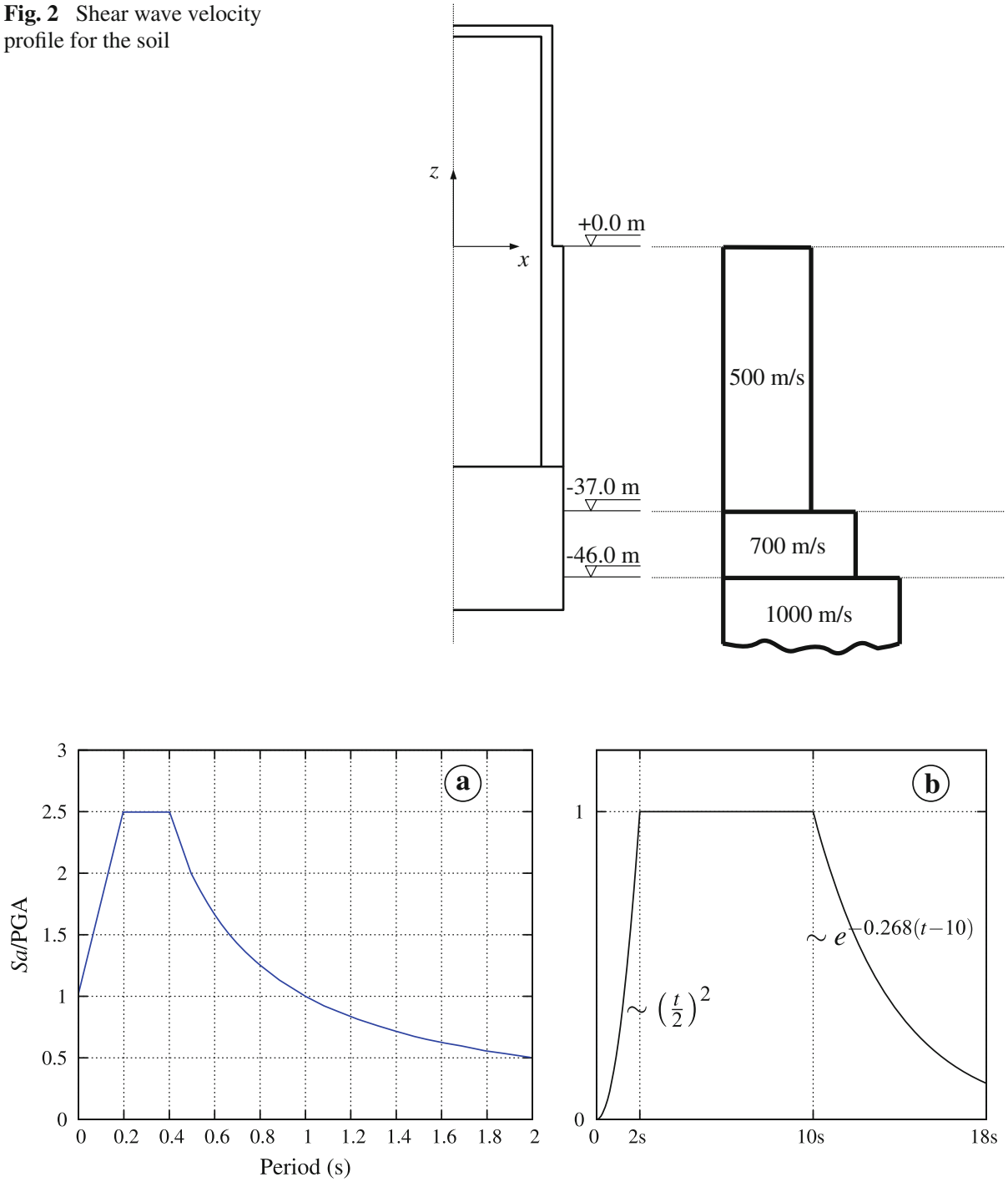

Fig. 3 a Design acceleration response espectrum, b Modulation scheme

As previously stated, the aim of the dynamic study is the assessment of the motion at discrete points of the structure consistent with a given free field ground motion. The latter has been defined in terms of the response spectrum shown in Fig. 3a, with a horizontal peak ground acceleration equal to $0.17 \mathrm{~g}$, and $10 \mathrm{~s}$ conventional duration, as defined by the client's specifications. In order to obtain some statistical significance, three different signals were generated using SIMQKE (Gasparini 1976) and then modulated using the scheme represented in Fig. 3b (Jennings et al. 1968). The resultant horizontal accelerograms are shown in Fig. 4, while the corresponding acceleration response spectra are presented in Fig. 5 together with the design acceleration response spectrum. 

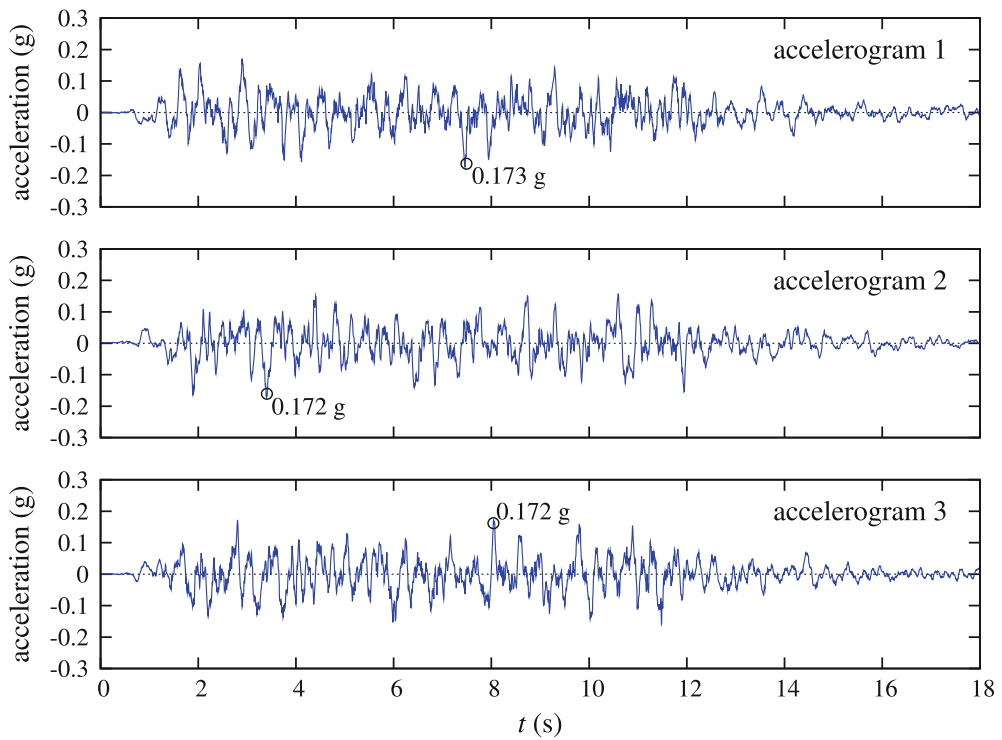

Fig. 4 Horizontal accelerograms

Fig. 5 Free-field elastic acceleration response spectra

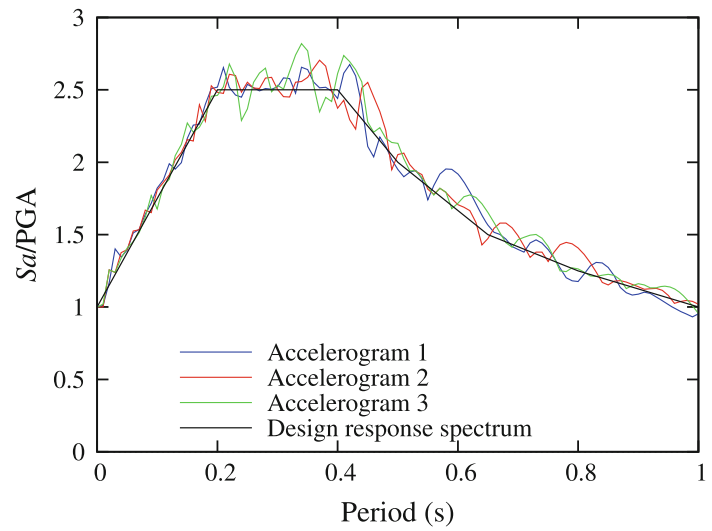

\section{Methodology}

Once geometry, stratigraphy and seismic action have been defined, the seismic response of the structure is going to be computed by using two methodologies: a three-step approach and a direct approach (c.f. Fig. 6), as briefly described next. The objective is to simultaneously obtain the motion at the surface and at discrete points of the structure, under vertically plane harmonic incident $S$ waves of fixed frequency. The complex valued relationships may be used to transform a given free field motion into the motion at the reference point, in the time domain.

Substructuring methods in the hypothesis of a perfectly rigid structure provide the response following a three-step procedure (Kausel and Roesset 1974; Kausel et al. 1978): (i) computation of the kinematic interaction factors; (ii) computation of stiffness and damping coefficients; and finally (iii) estimation of inertial interaction (see Fig. 6). 


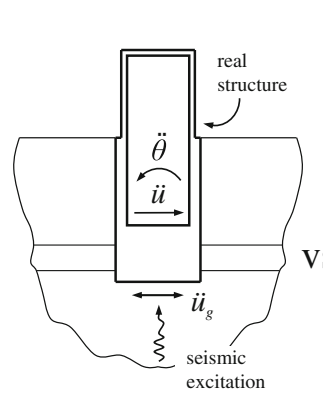

Total

Solution

$$
\uparrow
$$

Direct

Methods

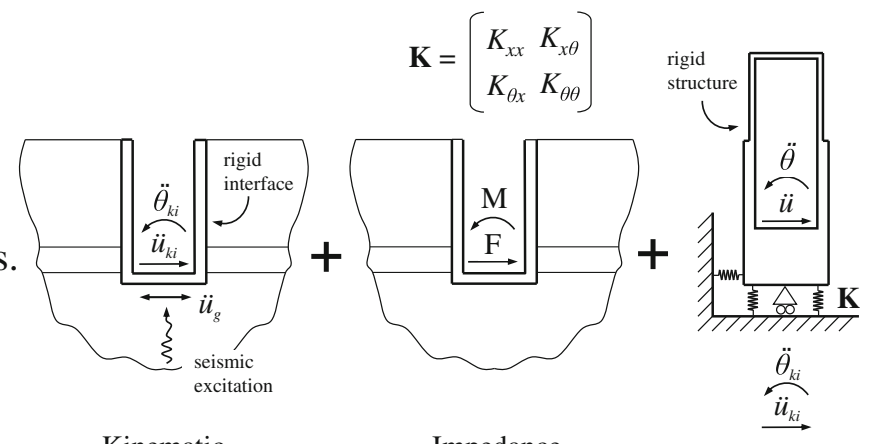

Impedance

Matrix of Soil
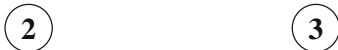

Fig. 6 Direct method versus three-step solution

The second alternative is the use of direct methods. They model all domains defining soil and structure simultaneously. Therefore they allow taking into account the interactions among the different elements in a more rigorous way. The more popular numerical techniques used in this approach are FEM and BEM. In this work, the Boundary Element Method (Domínguez 1993) has been used for the direct approach because it is specially suitable to deal with the dynamic analysis of unbounded domains such as the soil layers in this problem.

When applying the three-step method, simplified expressions available in the bibliography are usually used to estimate kinematic interaction factors and impedance functions (Mylonakis et al. 2006; Kausel et al. 1978). However, accurate enough expressions are not available for the layered soil profile of the problem at hand. For this reason, again BEM is also used to assess the kinematic interaction coefficients and the soil impedances corresponding to the first and second steps of the sub-structuring methodology.

When using the BEM to solve the problem following a direct approach, or in order to compute kinematic interaction factors and impedances for substructuring approach, all domains defining the geometry of the problem (soil layers and concrete walls) are modelled as linear homogeneous isotropic viscoelastic regions, and welded conditions are assumed between the different domains. Figures 7 and 16 show two boundary element meshes used for the sub-structuring problems (step 1 and 2) and direct approach respectively. It can be appreciated that the boundary element code allows the use of quadratic triangular (6 nodes) and quadrilateral ( 9 nodes) elements. This particular geometry also includes corner problems where an interface cuts the embedded structure. Here, corner problems are solved by means of a non-nodal collocation strategy, which also allows using non-conforming meshes, as can be seen in the figures. The element size must be smaller than the half-wave length at the corresponding region for the highest frequency of analysis, in this case $25 \mathrm{~Hz}$, although in general, all mesh parameters, such as free-surface extension and number of elements, are defined by performing convergence analyses of the variables of interest for different meshes. It is also worth noting that, even though the figure shows three quarters of the problem geometry, only a quarter of the total geometry is really meshed, as the code is able to take 
Fig. 7 Boundary elements mesh used for the kinematic interaction and soil impedance problems

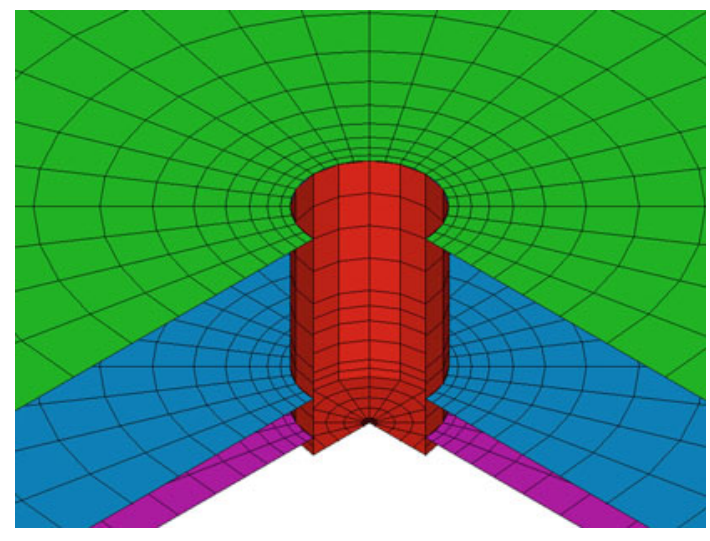

into account the symmetry properties of the problem. More details of the used boundary element code can be found in Maeso et al. (2002, 2004, 2005).

Next section provides the kinematic interaction coefficients, while the soil impedances are presented in Sect. 5. These results are later used in Sect. 6 to compute the dynamic response of the whole system by solving the inertial interaction step of the sub-structuring method. They are also interesting because they enable understanding the sensitivity of the final response to different characteristics of the system. Finally, and following a direct approach, the real problem is solved modelling all elements simultaneously in Sect. 7. Results are presented compared to those obtained through the three-step method.

\section{Kinematic interaction}

In this section, the displacements and rotations of the massless and infinitely rigid structure are obtained considering vertically incident $S$ waves. The problem is well known (Roesset 1977), as well as its approach through the boundary element method (Domínguez 1993; Álvarez Rubio et al. 2005). Figure 7 shows the boundary element mesh used for this problem. The free surface and the interfaces among strata are displayed with different colours. The rigid interface among soil and structure is depicted in red.

Figure 8 presents absolute values of the translational $I_{u}=u / u_{\mathrm{ff}}$ and rotational $I_{\Phi}=\theta R / u_{\mathrm{ff}}$ kinematic interaction factors of the problem at hand, being $u$ and $\theta$ the horizontal displacements and rotations at the base of the structure, $R$ the radius of the structure and $u_{\mathrm{ff}}$ the free-field horizontal motion at the ground surface. The horizontal axis represents the dimensionless frequency $a_{o}=\omega R / c_{S}$, being $\omega$ the angular frequency of the excitation and $c_{S}$ the velocity of the $\mathrm{S}$ waves in the top layer $(500 \mathrm{~m} / \mathrm{s})$. The figure presents, together with the results obtained using boundary elements, the transfer functions obtained through the simple rules proposed by Elsabee et al. (1977) for homogeneous soils (see also Kausel et al. (1978); Mylonakis et al. (2006)). The agreement between them is good at low and high non-dimensional frequencies, while discrepancies appear between 0.4 and 2.0. These differences would have a significant impact on the seismic assessment since the key components hosted by the structure have their fundamental frequencies within this range. Thus, only kinematic interaction factors obtained using BEM will be used in Sect. 6. 

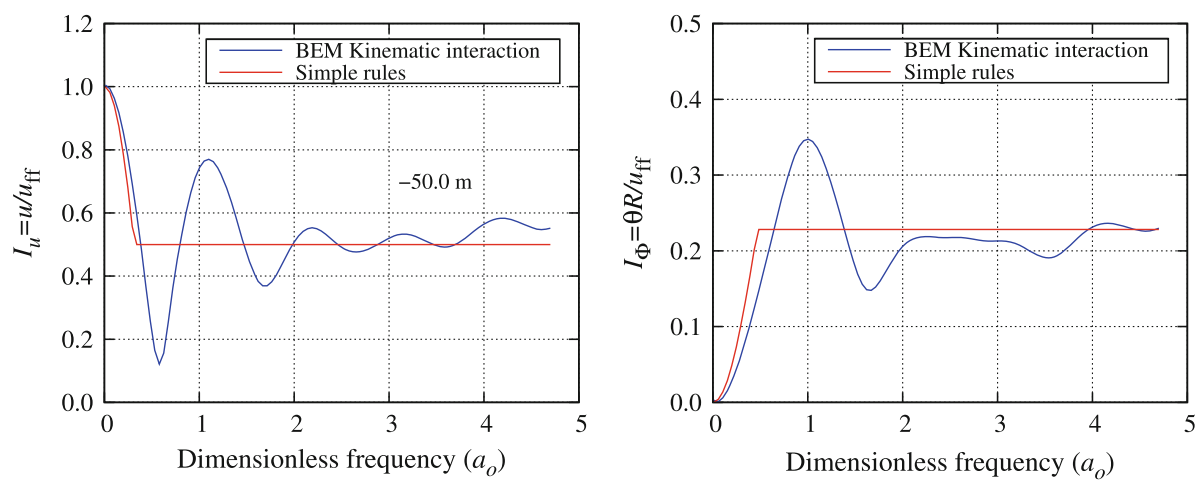

Fig. 8 Kinematic interaction factors at the base of the structure for vertically incident $\mathrm{S}$ waves

\section{Soil impedances. Equivalent springs and dashpots}

The second step in the three-step method is the determination of the soil impedances (c.f. Fig. 6). Impedance functions corresponding to a wide variety of cases can be found in the literature (Wolf 1985; Mylonakis et al. 2006). However, no such functions are available for this particular configuration. Therefore, in this work, horizontal and rocking stiffness and damping coefficients have been obtained using the above mentioned boundary element code by prescribing unitary harmonic rigid-body horizontal displacements or rotations at the soilstructure interface. For each harmonic frequency the impedances are obtained by integration of the relevant stresses in the interface. It is worth noting that rotations induce horizontal stresses in the cylinder walls and, reciprocally, horizontal motions produce a momentum at the base. Therefore, with reference to the central point at the base of the structure, horizontal and rocking impedances are coupled. The coupling term becomes more important the deeper the structure is embedded.

Impedance functions can then be arranged in a stiffness matrix $\mathbf{K}$ relating forces and moments $\mathbf{F}$ applied at the center of the base to the resulting displacements and rotations $\mathbf{u}$ in a relationship of the type $\mathbf{F}=\mathbf{K u}$ that condenses the dynamic properties of the soil profile and that can be written as

$$
\left\{\begin{array}{l}
F \\
M
\end{array}\right\}=\left[\begin{array}{ll}
K_{x x} & K_{x \theta} \\
K_{\theta x} & K_{\theta \theta}
\end{array}\right]\left\{\begin{array}{l}
u \\
\theta
\end{array}\right\}
$$

where each term $K_{l m}$ depends on the dimensionless frequency $a_{o}$ and represents the force (or moment) at the base of the structure needed to obtain a harmonic unitary displacement (or rotation). As forces and displacements are out of phase, terms $K_{l m}$ are complex valued, in the form

$$
K_{l m}=k_{l m}+i a_{o} c_{l m}
$$

where $k_{l m}$ and $c_{l m}$ are the stiffness and damping coefficients, respectively.

Figure 9 presents the stiffness and damping functions of the problem at hand, computed with the mesh shown in Fig. 7, and normalized using the shear stiffness of the top soil layer and the radius of the embedded structure. It is worth noticing that resonant frequencies associated to the shear deformation of the top two soil strata do not become apparent in these impedance functions. This is due to the fact that the related shear modes do not get excited 

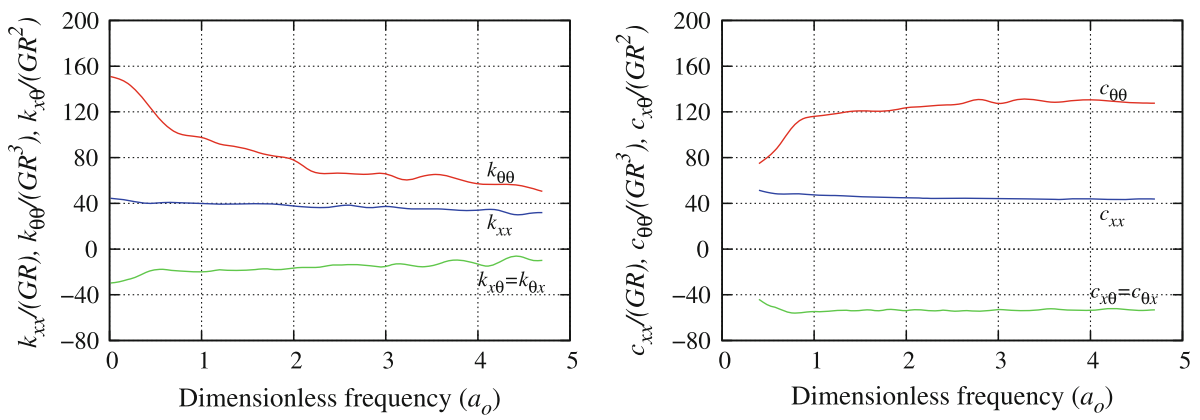

Fig. 9 Horizontal and rocking stiffness and damping functions

because the structure goes all the way through the soil layers and does not produce significant vertical $\mathrm{S}$ waves.

\section{Inertial interaction. Dynamic response of the structure.}

In previous steps, the impedance functions, that condense the dynamic properties of the soil profile taking into account the embedded structure, and the seismic excitation including the effects of kinematic interaction, have been assessed. In the third step, the dynamic response of the system is assessed under the assumption of the structure being infinitely rigid. Thus, the rigid body motion equations that govern the dynamic behavior of the structure can be written in the frequency domain as

$$
\begin{aligned}
& {\left[\begin{array}{ll}
K_{x x}-\omega^{2} M & K_{x \theta}+\omega^{2} M \overline{h_{g}} \\
K_{x x} \overline{h_{g}}+K_{\theta x} & K_{x \theta} \overline{h_{g}}+K_{\theta \theta}-\omega^{2} I_{G}
\end{array}\right]\left[\begin{array}{l}
u \\
\theta
\end{array}\right]=} \\
& {\left[\begin{array}{ll}
K_{x x} & K_{x \theta} \\
K_{x x} \overline{h_{g}}+K_{\theta x} & K_{x \theta} \overline{h_{g}}+K_{\theta \theta}
\end{array}\right]\left[\begin{array}{c}
u_{k i} \\
\theta_{k i}
\end{array}\right]}
\end{aligned}
$$

where $u$ and $\theta$ are the horizontal displacement and rotation at the base of the structure; and $u_{k i}$ and $\theta_{k i}$ are the input horizontal displacement and rotation due to the seismic excitation, which are obtained from the kinematic interaction factors. Equation 3 can be solved numerically for any given frequency to obtain the structural response of the rigid body in terms of its displacements $u$ and rotations $\theta$. This means that, once impedance functions and kinematic interactions factors have been computed, the cost of computing the system response is very low. This allows to perform parametric analyses and study the influence of different aspects on the final response at low cost, which is one key benefit of the three-step approach.

Figure 10 presents the dynamic response of the structure, computed making use of this sub-structuring methodology, as a function of the dimensionless frequency $a_{o}$. The translational kinematic interaction factors presented in Sect. 4 are also plotted in this figure in order to allow assessing the relative importance of kinematic interaction and inertial interaction on the final response of the system. In both cases, the transfer functions relate the horizontal displacement $u$ at the base of the structure to the free-field motion $u_{\mathrm{ff}}$ at the ground surface. Under the rigid body motion assumption, horizontal displacements at any point of the structure can be easily obtained, and results for six different depths are presented. The results presented in Fig. 10 indicate that the presence of the structure filters out an important part of the seismic signal, mainly for frequencies $a_{o} \geq 2$. In order to illustrate the effects of this 

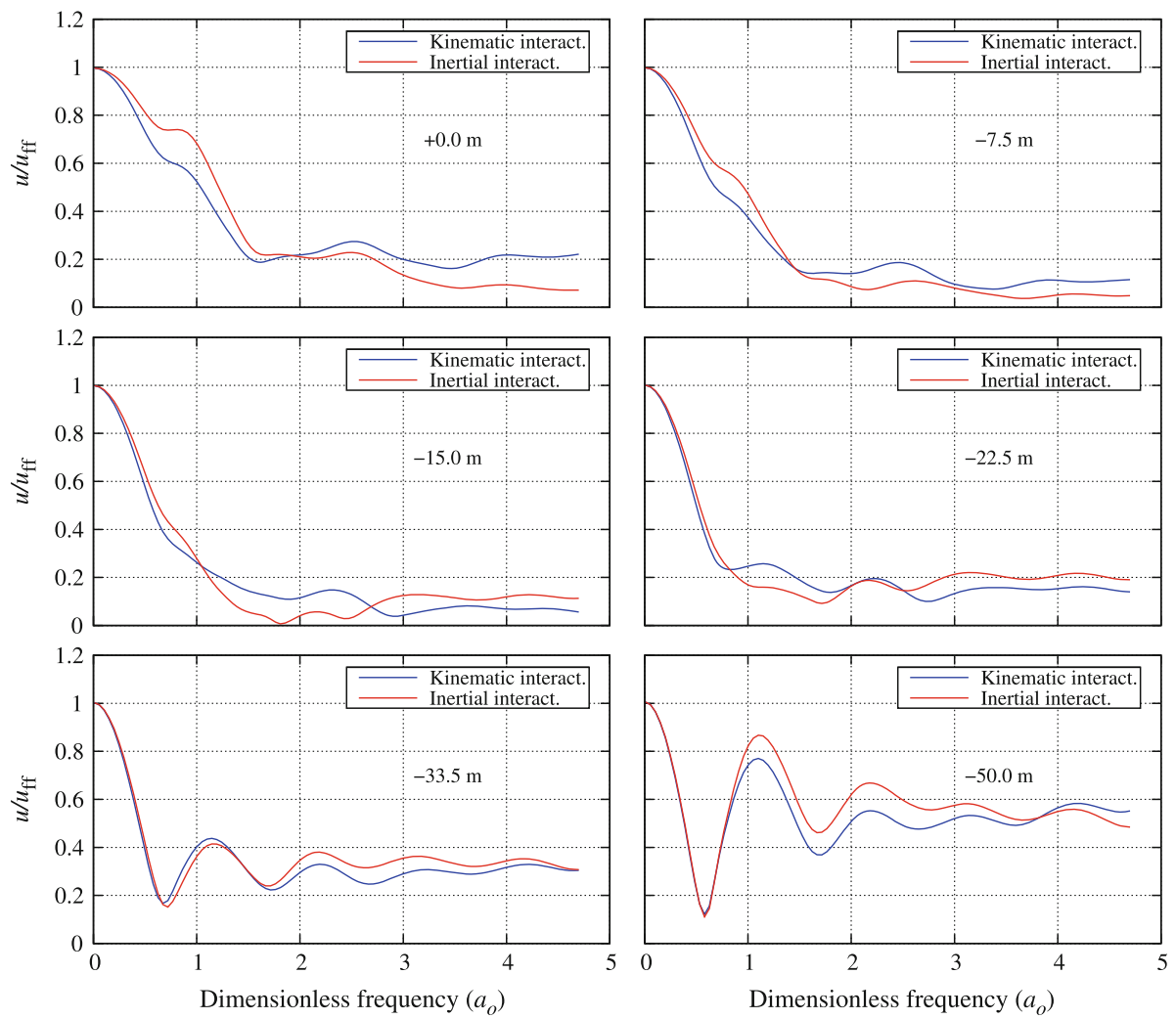

Fig. 10 Frequency response functions for the normalized horizontal displacements from inertial interaction analysis and translational kinematic interaction factors for vertically incident $\mathrm{S}$ waves

filtering, Fig. 11 shows acceleration records evaluated on the cylinder at depths 0 and $-50 \mathrm{~m}$ when the system is subjected to the first design accelerogram (c.f. Fig. 4). These responses have been computed as the inverse Fourier transform of the product between the frequency response function obtained for step 3 (inertial interaction) and the discrete Fourier transform of accelerogram 1, using the Fast Fourier Transform algorithm. The free-field accelerogram 1 is also presented in Fig. 11 in order to provide a baseline. The input seismic signal is filtered by the presence of the structure, as the peak acceleration is reduced from 0.173 to $0.087 \mathrm{~g}$ $(\sim-50 \%)$ at $-50.0 \mathrm{~m}$ depth, while the rotation induced by the incident wave-field increases the acceleration to $0.114 \mathrm{~g}$ at $0.0 \mathrm{~m}$. The latter acceleration value is still $35 \%$ smaller than the free field peak acceleration of the input seismic signal.

Elastic response spectra can also be obtained using these filtered signals at different depths as the excitation of one degree-of-freedom systems. Figure 12 presents the pseudoacceleration response spectra corresponding to the inertial interaction (step 3) and only kinematic interaction (step 1) for different depths. The design response spectra and the ones corresponding to the input seismic signals at free-field surface $(+0.0 \mathrm{~m})$ are also presented for reference in all plots. All results are shown in terms of envelopes for the three input accelerograms presented in Fig. 4.

Figures 10 and 12 also show that results obtained from step 3 (inertial interaction) or directly from step 1 (kinematic interaction) agree extremely well, which means that kinematic 

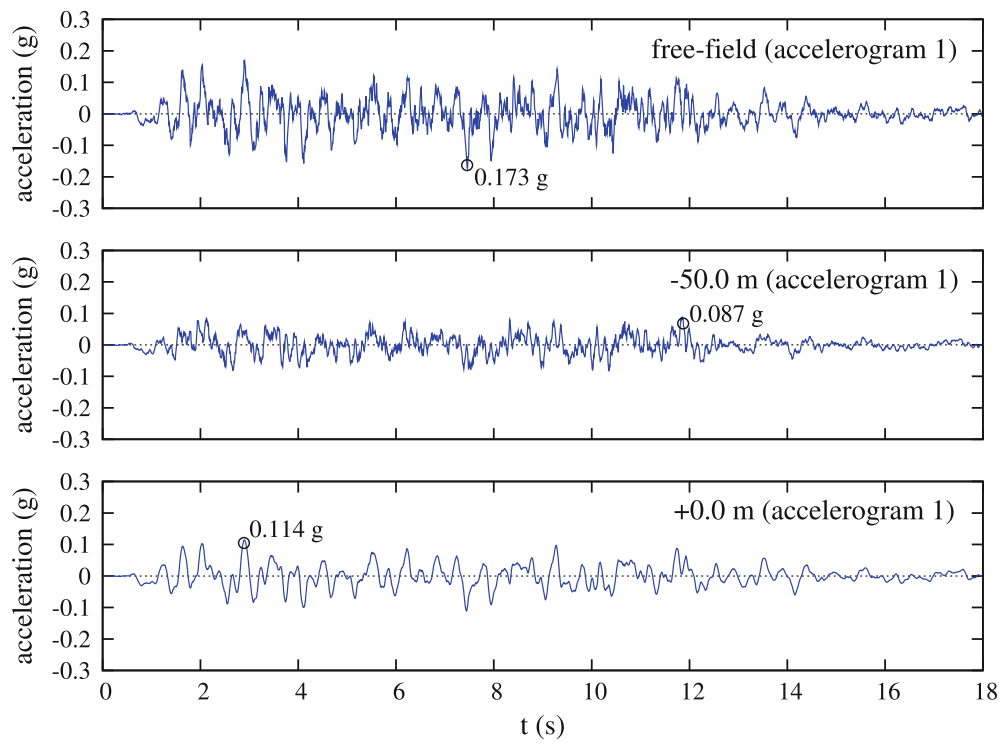

Fig. 11 Structural response at different depths according to the three-step solution for accelerogram 1

interaction dominates the dynamic response of this structure. In order to understand this effect, Eq. 3 has been rewritten with $\mathbf{u}=\mathbf{u}_{k i}+\overline{\mathbf{u}}$, being $\mathbf{u}_{k i}$ the vector of displacements and rotation at the base of the rigid foundation in the kinematic interaction problem, and $\overline{\mathbf{u}}$ the vector of relative displacements and rotations between soil and structure. This relative motions, which are responsible for the differences between inertial and kinematic interaction, happen to be relatively small in the frequency range of interest. They are also closely related to the damping properties of the system, which can be studied in terms of the damping factors $\zeta$. These factors can be estimated both for the horizontal and rocking vibrations from the corresponding impedance functions as

$$
\begin{array}{r}
\zeta_{x}=\frac{\tilde{c}_{x x}}{2 \sqrt{M k_{x x}}} ; \quad \tilde{c}_{x x}=\frac{R}{c_{s 1}} c_{x x} \\
\zeta_{\theta}=\frac{\tilde{c}_{\theta \theta}}{2 \sqrt{\left(I_{G}+M h_{g}^{2}\right) k_{\theta \theta}}} ; \quad \tilde{c}_{\theta \theta}=\frac{R}{c_{s 1}} c_{\theta \theta}
\end{array}
$$

As shown in Fig. 13, where this damping factors are plotted as a function of the dimensionless frequency, the horizontal vibration is overcritically damped while the rocking vibration is undercritically damped for $a_{o}<2$. This may explain why the system response is dominated by kinematic interaction, and also why the largest differences are observed for points near the surface, where the effect of the structural rotation is larger.

The fact that inertial interaction is so close to the corresponding kinematic interaction factors is an extremely appealing idea, as it could imply that, in certain cases, kinematic interaction itself can be a reasonably good approximation of the structural response and, therefore, the computation of impedance functions and inertial interaction could be avoided. For this reason, the key factors that determine whether this simplification can be carried out will be briefly investigated next. 

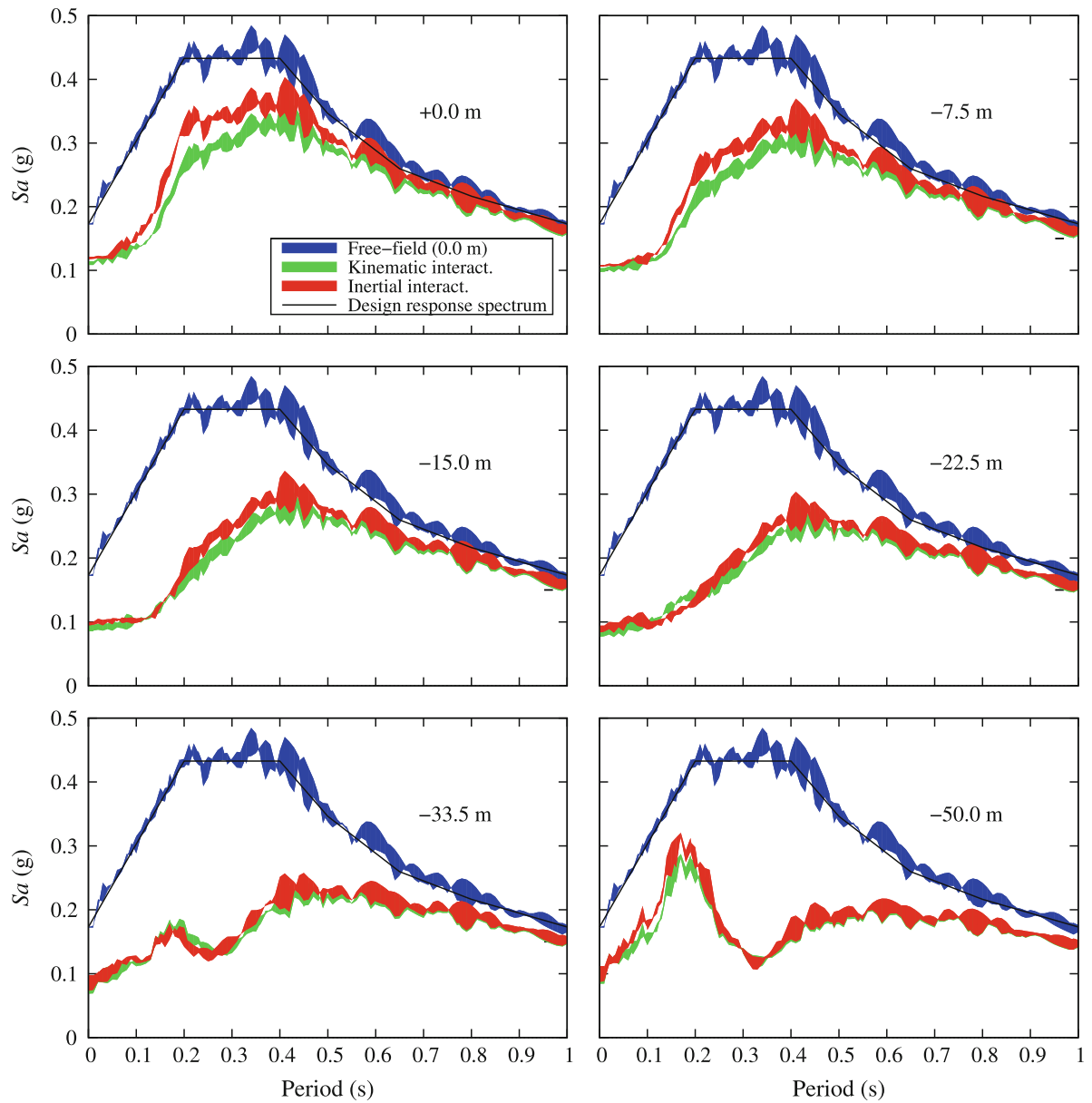

Fig. 12 Envelopes of pseudo-acceleration response spectra at different depths

Fig. 13 Damping factors for the horizontal and rocking modes

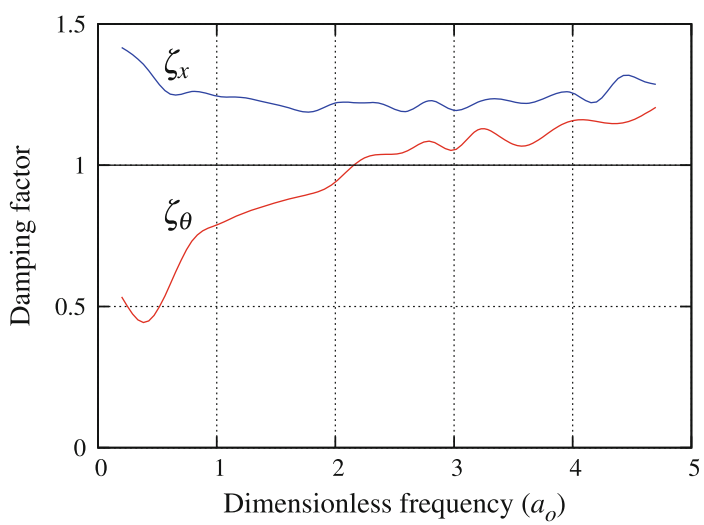



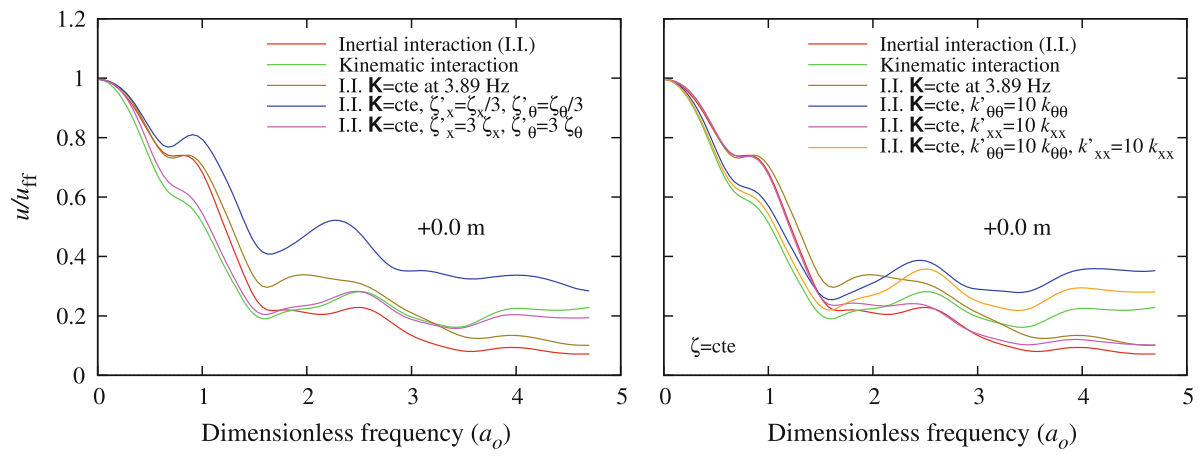

Fig. 14 Frequency response functions for different damping factors (left) and natural frequencies (right)
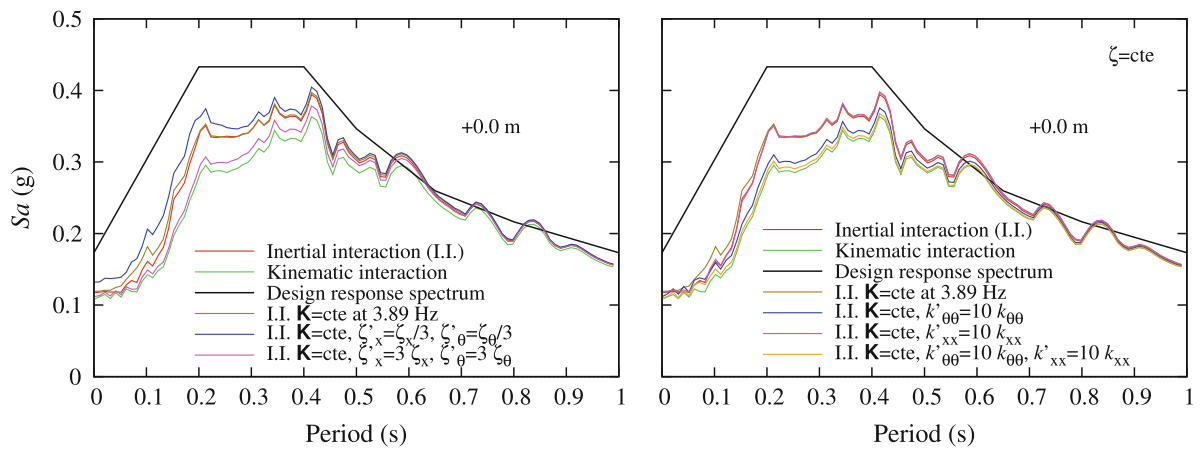

Fig. 15 Pseudo-acceleration response spectra for different damping factors (left) and natural frequencies (right)

In order to be able to concentrate on a few key parameters, and taking into account that the impedance functions (excepting the rocking one) are approximately frequency-independent, a slightly simplified system with stiffness and damping coefficients being frequency independent will be used. These constant values are chosen as those corresponding to $a_{o}=0.74$. This is a central frequency in the range $0<a_{o}<1.5$ of interest, and also the natural frequency of one of the sensitive devices hosted by the structure. For $a_{o}=0.74$, the constant stiffness and damping coefficient values are: $k_{x x} / G R=39.0, k_{\theta \theta} / G R^{3}=104.2, k_{x \theta} / G R^{2}=-17.3$, $c_{x x} / G R=43.8, c_{\theta \theta} / G R^{3}=96.9$ and $c_{x \theta} / G R^{2}=-49.6$. Figures 14 and 15 present the frequency response function and the corresponding elastic response spectrum for such constant impedances. It can be seen that, in the frequency range of interest, the system response is very close to the one obtained with a more rigorous inertial interaction response (i.e. with frequency dependent impedances).

In order to investigate if damping factors are responsible for the dynamic response of the system being dominated by kinematic interaction factors a parametric study has been performed. The left plot of Fig. 14 presents the frequency response functions corresponding to systems where the damping coefficients have been artificially modified by factors 3 and 1/3. The left plot of Fig. 15 presents the corresponding acceleration response spectra. As expected, when damping factors are significantly smaller than one, the system response becomes different from the one considering kinematic interaction factors alone. On the contrary, for 
Table 2 System natural frequencies for different configurations

\begin{tabular}{llr}
\hline & $a_{o_{1}}\left[f_{1}(\mathrm{~Hz})\right]$ & $a_{o_{2}}\left[f_{2}(\mathrm{~Hz})\right]$ \\
\hline$k_{x x}, k_{\theta \theta}$ & $1.3[7.0]$ & $3.2[17.0]$ \\
$k_{x x}, 10 k_{\theta \theta}$ & $2.0[10.6]$ & $7.0[37.0]$ \\
$10 k_{x x}, k_{\theta \theta}$ & $1.4[7.3]$ & $10.2[53.9]$ \\
$10 k_{x x}, 10 k_{\theta \theta}$ & $3.8[20.4]$ & $11.4[60.6]$ \\
\hline
\end{tabular}

very large damping factors, both transfer function and response spectrum get very close to it.

Aside from the damping factor, the other dynamic properties that have a strong influence on the dynamic response of the system are its natural frequencies. Table 2 presents the undamped natural frequencies of the coupled system for the stiffness matrix corresponding to $a_{o}=0.74$. Also three more combinations are considered in which the horizontal and rocking stiffnesses are alternatively or simultaneously multiplied by a factor of 10 , in order to artificially increase the natural frequencies of the system and take them out of the frequency range that contains most of the energy of the design accelerograms $\left(0<a_{o}<1.5\right)$. It is worth noting that, in the original configuration, but also when the horizontal stiffness is multiplied by a factor of 10 , the first natural frequency is inside this range. On the contrary, when the rocking stiffness is increased, both natural frequencies are well above $a_{o}=1.5$. The effects of these changes are reflected in the right plots of Figs. 14 and 15, that present the corresponding frequency response functions and response spectra. In this plots, both damping factors $\zeta_{x}$ and $\zeta_{\theta}$ have been deliberately kept constant. It can be seen that when only the horizontal stiffness is artificially increased, the system inertial response remains almost unchanged. On the contrary, when the rocking stiffness is increased, the system response gets really close to the kinematic interaction problem. This is due to the fact that, when only the horizontal stiffness is changed, the first natural frequency is only slightly modified, and the rotation of the structure (with a great influence on the motions at $+0.0 \mathrm{~m}$ ) is not significantly altered. It can be concluded that it is the rotational behavior of the soil-structure system what drives the final dynamic response.

\section{Direct method}

As said before, the three-step approach implies the assumption of a perfectly rigid structure. On the contrary, the direct approach allows a more rigorous analysis that takes into account the true flexibility of the structure in order to obtain the coupled response of the system subjected to the seismic excitation. In this case, soil and structure are analysed simultaneously under the action of vertically incident $\mathrm{S}$ waves using the boundary element code presented above and the mesh shown in Fig. 16.

Figure 17 presents the frequency response functions for the normalized horizontal displacements of different points of the structure. Results obtained from the direct approach and taking into account the true flexibility of the structure are labeled as 'Flexible model', results corresponding to the three-step approach (already presented before) are labeled as 'Rigid model', and results obtained from the kinematic interaction problem are labeled as 'Kinematic interaction'. It can be seen that, in general, the assumption of a structure that behaves as a rigid body produces unsatisfactory results for all the frequency range, though acceptable results are obtained in some cases (mainly for high frequencies and at the base 

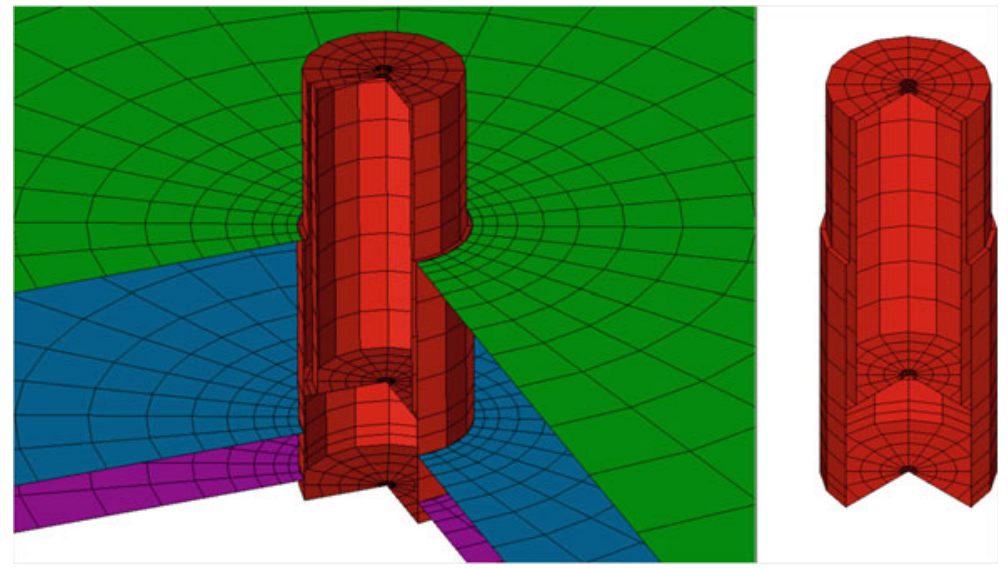

Fig. 16 Boundary elements mesh used for the direct approach. Flexible structure embedded in an stratified soil
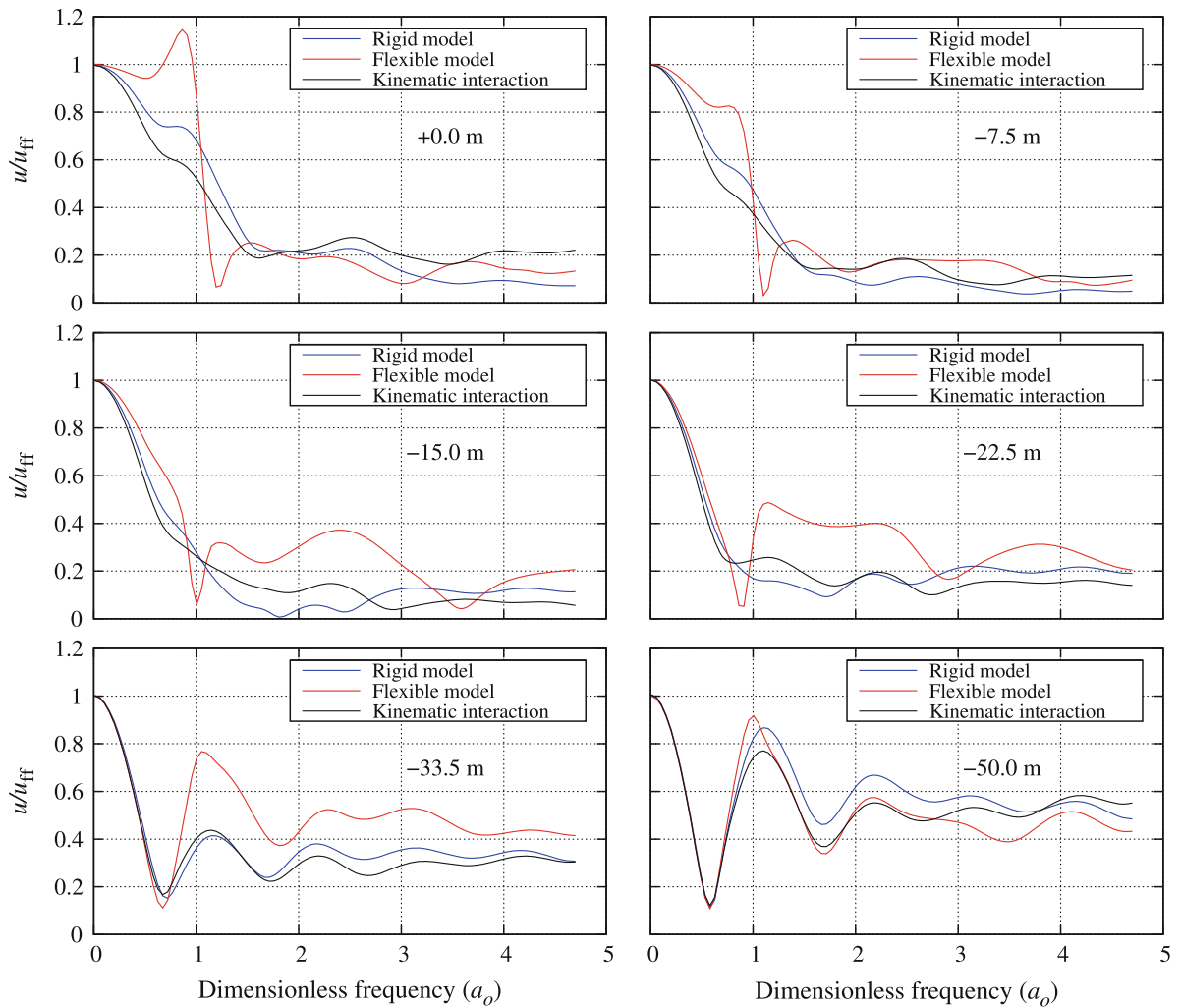

Fig. 17 Frequency response functions for the normalized horizontal displacements of different points of the structure under vertically incident $\mathrm{S}$ waves. Three-step and direct approaches

of the structure). As expected, the effects of the flexibility of the structure become more important with height. It is worth noting that the most important differences are found near the ground surface and below $a_{o}=1.5$, while the frequency range of interest in terms 

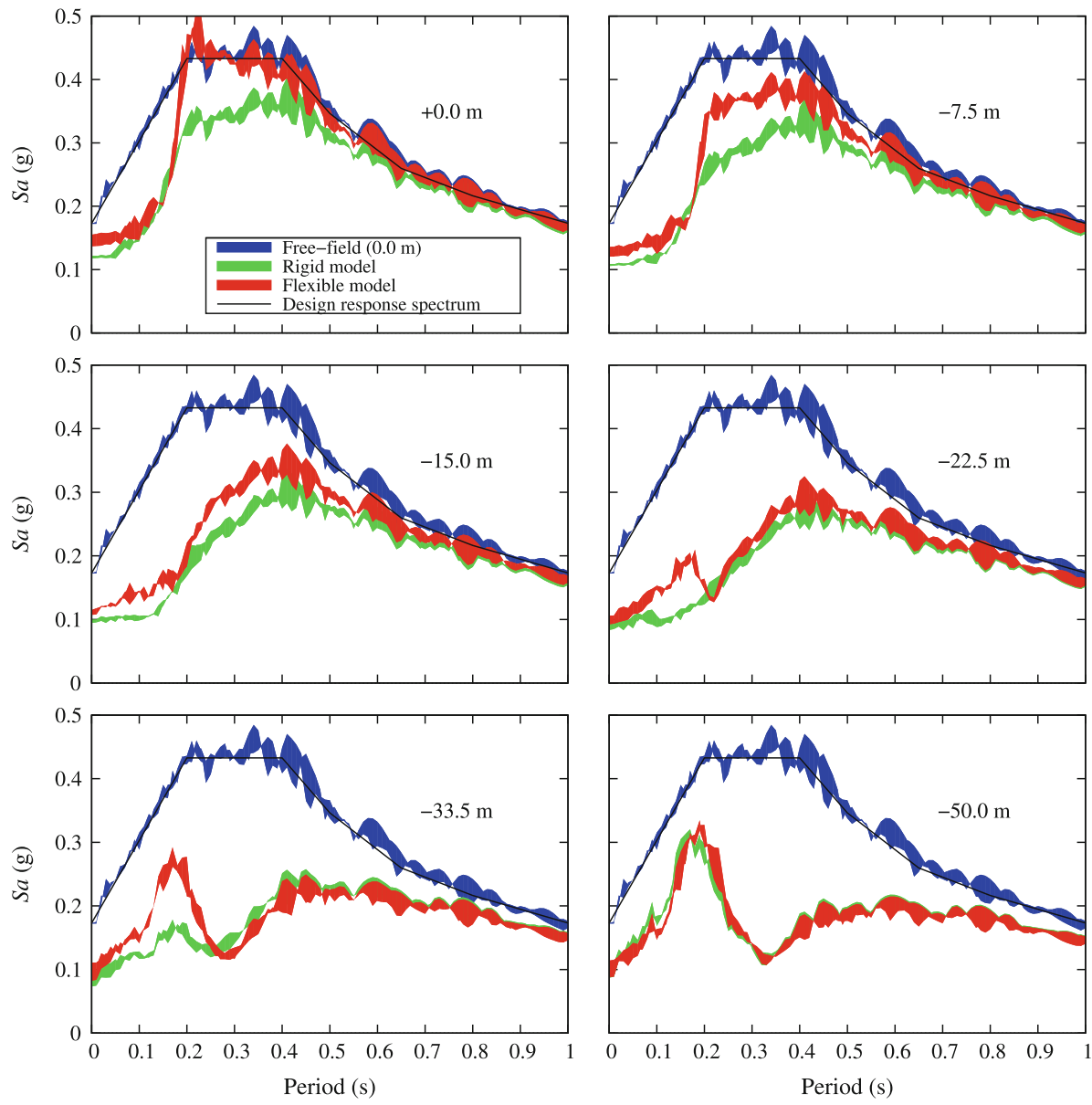

Fig. 18 Pseudo-acceleration response spectra at different levels obtained from the direct methodology (flexible model) and three-step solution (rigid model) methodologies

of the frequency content of the design accelerograms is also for $0<a_{o}<1.5$, thus affecting almost the whole frequency content of the input ground motions.

The influence of these differences between rigid and flexible models on the seismic response of the structure can be evaluated by means of elastic response spectra. To this end, Fig. 18 presents the envelopes of the pseudo-acceleration response spectra corresponding to the three design accelerograms in both rigid and flexible models. It can be seen that the response spectra for the rigid and flexible hypothesis are almost coincident at the base of the structure $(-50.0 \mathrm{~m})$, but remarkable differences can be appreciated for all other points. In this sense, even at $-33.5 \mathrm{~m}$ depth (a point belonging to the filled bottom part of the structure), differences are important in the range of periods between 0.1 and $0.25 \mathrm{~s}$. For example, the response computed for $T=0.17 \mathrm{~s}$ is $0.18 \mathrm{~g}$ for the rigid model and $0.29 \mathrm{~g}$ for the flexible model (approximately $60 \%$ difference).

The response of the structure at ground surface level $(+0.0 \mathrm{~m})$ is particularly interesting. At this level, and for periods higher than $0.2 \mathrm{~s}$, the presence of the flexible structure does not filter the input seismic signal, in contrast with the results obtained under the rigid structure 
Fig. 19 Validation of the hypothesis of rigidity of the structure. Maximum accelerations with depth under accelerogram 1 . Vertically incident $\mathrm{S}$ waves

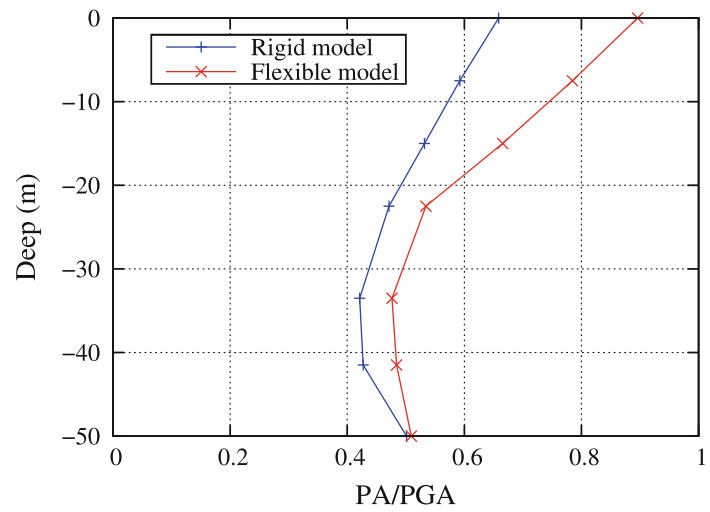

Fig. 20 Validation of the hypothesis of rigidity of the structure. Maximum normal stresses with depth under accelerogram 1 . Vertically incident $\mathrm{S}$ waves

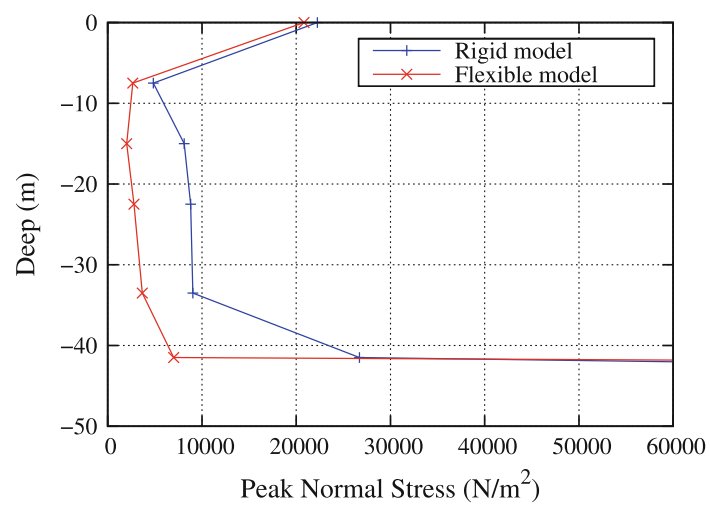

assumption. For instance, for $T=0.26 \mathrm{~s}$ (corresponding to $f=3.86 \mathrm{~Hz}$, one of the frequencies of interest) the spectral accelerations are $0.32 \mathrm{~g}$ for the rigid model and $0.46 \mathrm{~g}$ for the flexible model, implying a $44 \%$ difference.

The seismic response of the structure can be considered to result from the addition of three components: two rigid body motions (rotations and horizontal displacements) present in both methodologies; and a third one associated only with the flexural deformation of the structure. Results presented in Fig. 18 imply that this last component cannot be neglected in this particular problem, even if it is a large non-slender strongly embedded structure (Elsabee et al. 1977; Saitoh and Watanabe 2004), and therefore, the rigid body assumption is not valid in this case.

The same conclusions can be drawn from the analysis of the envelopes of maximum accelerations and normal stresses along the embedded part of the structure, comparing results from both the rigid and flexible models. Maximum accelerations are shown in Fig. 19, normalized with the peak ground acceleration (PGA), while peak normal stresses are represented in Fig. 20, both under design accelerogram 1. In both cases, the stresses have been computed with the boundary element method, using appropriate properties for each model. It is worth noting that results obtained making use of the rigid assumption underestimate maximum accelerations by up to $25 \%$. Considering normal stresses (c.f. Fig. 20), the flexibility of the structure implies a significant relaxation of the normal stresses across the soil-structure interface. 


\section{Conclusions}

The seismic analysis of sensitive equipment hosted by a non-slender and deeply embedded structure has motivated the work presented in this paper. The demand at different points of the structure has been obtained in terms of displacement frequency response functions and acceleration elastic response spectra. The main objective was to characterize the demand at different points of the structure in order to know if the assumption of infinite rigidity of the structure allows to obtain reliable values of its response.

To this end, the seismic response of the structure has been analysed using the sub-structuring (three-step solution) and direct methodologies. Both allow to take into consideration soil-structure interaction, which is known to be important in problems like the one considered here, but the three-step methodology assumes a perfectly rigid structure, while the direct one is able to handle its real flexibility.

As expected, demand has been found to be reduced by the presence of the structure. However, quite surprisingly, predictions using both methods show significant discrepancies. The assumption of a structure that behaves as a rigid body provides significant underestimations of the spectral accelerations at different points, with differences of up to $60 \%$, implying that the flexural deformation of the structure cannot be neglected in this particular problem and, therefore, that the structure cannot be considered to be a rigid one.

Also, the sub-structuring analysis have been useful to understand the key role of damping factors and rocking stiffness on the seismic response of the structure. It has been shown that, when damping factors are high, kinematic interaction is an extremely important element in the estimation of the structural response, and could even be used as a very good approximation to the inertial interaction step. This approximation will be even better if the natural frequencies of the system do not lie within frequency content of the seismic excitation.

Acknowledgments This work was supported by the Subdirección General de Proyectos de Investigación of the Ministerio de Economía y Competitividad (MINECO) of Spain and FEDER through research project BIA2010-21399-C02-01 and also by the Agencia Canaria de Investigación, Innovación y Sociedad de la Información (ACIISI) of the Government of the Canary Islands and FEDER through research project ProID20100224. A. Santana is recipient of the FPI research fellowship BES-2009-029161 from the MINECO. The authors would like to thank the engineer Mr. Elías Fernández who, by describing the problem as well as his concerns on the applicability of the current methods to solve it, motivated the research presented in the paper.

\section{References}

Álvarez Rubio S, Benito JJ, Sánchez-Sesma FJ, Alarcón E (2005) The use of direct boundary element method for gaining insight into complex seismic response. Comput Struct 83:821-835

Domínguez J (1993) Boundary elements in dynamics. Computational Mechanics Publication: Southampton and Elsevier Applied Science, New York

Elsabee F, Morray JP, Roesset JM (1977) Dynamic behavior of embedded foundations. Rep. No. R77-33, Massachusetts Institute of Technology, Cambridge

Gasparini DA (1976) SIMQKE: a program for artificial generation. Rep. No. R76-4. Massachusetts Institute of Technology, Cambridge

Ishihara K (1996) Soil behaviour in earthquake geotechnics. Oxford Science Publications, Oxford

Jennings PC, Housner GW, Tsai NC (1968) Simulated earthquake motions. Caeltech

Kausel E, Roesset JM (1974) Soil-structure interaction problems for nuclear containment structures. In: Electronic power and civil engineer conf paper power div specially conf, Boulder, Colorado, pp 469-498

Kausel E, Whitman RV, Morray JP, Elsabee F (1978) The spring method for embedded foundations. Nucl Eng Des 48:377-392 
Kausel E (2010) Early history of soil-structure interaction. Soil Dyn Earthquake Eng 30(9):822-832

Maeso O, Aznárez JJ, Domínguez J (2002) Effects of space distribution of excitation on seismic response of arch dams. J Eng Mech ASCE 128(7):759-768

Maeso O, Aznárez JJ, Domínguez J (2004) Three-dimensional models of reservoir sediment and effects on the seismic response of arch dams. Earthquake Eng Struct Dyn 33:1103-1123

Maeso O, Aznárez JJ, García F (2005) Dynamic impedances of piles and groups of piles in saturated soils. Comput Struct 83:769-782

Mylonakis G (2001) Elastodynamic model for large-diameter end-bearing shafts. Soil Found 41(3):31-44

Mylonakis G, Nikolaou S, Gazetas G (2006) Footings under seismic loading: analysis and design issues with emphasis on bridge foundations. Soil Dyn Earthquake Eng 26:824-853

Roesset JM (1977) Chapter 19: soil amplification of earthquakes. In: Desai CS, Christian JT (eds) Numerical methods in geotechnical engineering. McGraw-Hill, New York

Saitoh M, Watanabe H (2004) Effects of flexibility on rocking impedance of deeply embedded foundation. J Geotech Geoenviron Eng 130(4):435-445

Schabel B, Lysmer J, Seed HB (1972) SHAKE: a computer program for analysis of horizontally layered sites. Rep. No. EERC/72-12, University of California, Berkeley

Seed HB, Idriss IM (1970) Soil moduli and damping factors for dynamic response analysis. Report No 70-1. EERC

Varun D, Assimaki D, Gazetas G (2009) A simplified model for lateral response of large diameter caisson foundations: linear elastic formulation. Soil Dyn Earthquake Eng 29(2):268-291

Villaverde R (2009) Fundamental concepts of earthquake engineering. CRC Press, Taylor \& Francis, Baton Raton

Wolf JP (1985) Dynamic soil-structure interaction. Prentice Hall, Englewood Cliffs 